\title{
Feira interativa: Utilização de metodologias inovativas na educação em saúde
}

\author{
Interactive fair: Use of innovative methodologies in health education \\ Feria interactiva: Uso de metodologías innovadoras en educación para la salud
}

\begin{abstract}
Resumo
Uma feira em saúde se estabelece como instrumento no processo de aprendizagem, onde permite-se experienciar metodologias criativas diversas a partir de um espaço que possibilita explorar e compartilhar conhecimentos. Neste estudo, relata-se experiências de duas edições (dezembro/2018 e março/2019) de uma feira interativa como tecnologia em saúde, vivenciadas por discentes voluntários e bolsistas, inseridos em programas universitários de extensão e iniciação tecnológica. Foram realizadas em um Centro de Ensino Superior, divididas em quatro estações sequenciais com o público interagindo entre elas. Para as dinâmicas foram utilizados microscópios, lâminas, cartazes, maquetes e jogo de tabuleiro. Na primeira estação, foram caracterizados os parasitos mais prevalentes no Centro (a partir de pesquisa prévia), com visualização de formas parasitárias; na segunda, foi apresentada sintomatologia das enteroparasitoses em questão; na terceira, maquetes com os ciclos parasitários envolvidos, mecanismos de infecção e meios de prevenção. Já a quarta, foi um jogo de tabuleiro contendo perguntas contextualizadas das estações anteriores. Foi utilizado um quiz, com perguntas convertidas em um jogo com pontuação, interação e ranqueamento (acompanhamento dos conhecimentos pré e pós percurso). Com as feiras foi perceptivo observar que ações de educação em saúde, quando bem planejadas, surtem efeitos significativos e alcançam os objetivos de suas realizações. Palavras-chave: Tecnologias em saúde; Promoção da saúde; Doenças parasitárias.
\end{abstract}




\begin{abstract}
A health fair is established as an instrument in the learning process, where it is possible to experience different creative methodologies from a space that makes it possible to explore and share knowledge. In this study, experiences from two editions (December / 2018 and March / 2019) of an interactive fair as technology in health, experienced by volunteer and scholarship students, inserted in university programs of extension and technological initiation are reported. They were held in a Higher Education Center, divided into four sequential stations with the public interacting between them. For the dynamics, microscopes, slides, posters, models and board games were used. In the first season, the most prevalent parasites in the Center (from previous research) were characterized, with visualization of parasitic forms; in the second, symptoms of the enteroparasitoses in question were presented; in the third, models with the parasitic cycles involved, mechanisms of infection and means of prevention. The fourth, on the other hand, was a board game containing contextualized questions from previous seasons. A quiz was used, with questions converted into a game with punctuation, interaction and ranking (monitoring of pre and post course knowledge). With the fairs, it was perceptible to observe that health education actions, when well planned, have significant effects and achieve the goals of their achievements.
\end{abstract}

Keywords: Health technologies; Health promotion; Parasitic diseases.

\title{
Resumen
}

Una feria de la salud se establece como un instrumento en el proceso de aprendizaje, donde es posible experimentar diferentes metodologías creativas desde un espacio que permite explorar y compartir conocimientos. En este estudio se reportan experiencias de dos ediciones (diciembre / 2018 y marzo / 2019) de una feria interactiva como tecnología en salud, vivida por estudiantes voluntarios y becarios, insertos en programas universitarios de extensión e iniciación tecnológica. Se llevaron a cabo en un Centro de Educación Superior, dividido en cuatro estaciones secuenciales con el público interactuando entre ellas. Para la dinámica se utilizaron microscopios, diapositivas, carteles, maquetas y juegos de mesa. En la primera temporada se caracterizaron los parásitos más prevalentes en el Centro (a partir de investigaciones previas), con visualización de formas parasitarias; en el segundo se presentó la sintomatología de las enteroparasitosis en cuestión; en el tercero, modelos con los ciclos parasitarios implicados, mecanismos de infección y medios de prevención. El cuarto, en cambio, era un juego de mesa que contenía preguntas contextualizadas de temporadas anteriores. Se utilizó un cuestionario, con preguntas convertidas en un juego con puntuación, interacción y ranking (seguimiento de los conocimientos previos y posteriores al curso). Con las ferias fue perceptible observar que las acciones de educación en salud, bien planificadas, tienen efectos significativos y logran las metas de sus logros.

Palabras clave: Tecnologías sanitarias; Promoción de la salud; Enfermedades parasitarias.

\section{Introdução}

A educação em saúde, como discutido em Salci et al. (2013, p. 224), objetiva a sensibilização, conscientização e mobilização individuais e coletivas para o enfrentamento de situações que interferem na qualidade de vida dos sujeitos, utilizando-se de práticas pedagógicas de cunho participativo e emancipatório (Brasil, 2009; Matta, 2009). Ela não deve ser reduzida a prática de transmitir informações sobre saúde, mas, ser considerada como meio de promoção da saúde. Para isso se faz necessário que a mesma possa estimular a compreensão do tema, conceitos e dos aspectos abordados, associada à qualidade de informação, comunicação efetiva e escuta qualificada, para um processo educativo mais palpável.

Tecnologias educativas para a promoção da saúde utilizando-se de metodologias ativas (ou ino-vativas), não servem apenas como fonte de motivação, mas também como forma de construção de conhecimento, constituindo-se em ferramentas que ensinam enquanto divertem, estimulam a autoaprendizagem e despertam a curiosidade (Filatro \& Cavalcanti, 2018; Maike, 2013).

Dentre as ações promovidas no processo de educação em saúde, a feira de saúde constitui-se como meio elementar, visto que pode ser constituída das mais variadas metodologias, com efeitos positivos para a conscientização da temática abordada. Uma vez que, possibilita aos envolvidos experienciar o método científico experimental, além de incentivar a interação ativa junto à comunidade trabalhada, atentando-se sempre para as necessidades da mesma.

A feira de saúde pode assim ser considerada uma tecnologia educativa no processo de aprendizagem, em que é permitido ao discente experienciar o processo de organização, criatividade na elaboração das metodologias, oportunizando-os 
a desenvolverem a oralidade. E aos ouvintes é oferecido um espaço de interação, permitindo-se investigar, explorar, expor experiências e compartilhar conhecimento.

Um ponto de partida ao trabalhar com as feiras de saúde é a ideia de oferecer uma prática interdisciplinar e interprofissional, rompendo com o ensino fragmentado e linear, restrito à experiência de sala de aula, e buscando propiciar experiências em que os discentes percebam como o conhecimento depende do saber de distintas áreas e que pudessem trabalhar em um cotidiano que enfatizasse o senso de autonomia, bem como fossem expostos a situações concretas da realidade de saú de do país e da região (Ferreira et al., 2010). Atividades de ensino, pesquisa e extensão nos moldes de uma Feira de Saúde podem contribuir, e muito, com a formação de um profissional mais humano, sensível às necessidades das pessoas ao seu redor, mais reflexivo, proativo, crítico e com senso de responsabilidade social (Tavares, Costa, Ninahuaman, Marques \& Santos, 2017).

Nessa perspectiva, a extensão pode ser utilizada como meio de propagar boas práticas de educação em saúde. Assim, o objetivo do presente trabalho, é descrever um relato de experiências vividas por discentes voluntários e bolsistas dos Programas Institucionais de Bolsa de Extensão Universitária (PIBEX) e de Bolsa de Iniciação em Desenvolvimento Tecnológico e Inovação (PIBITI), promovidos, respectivamente, pela Pró-Reitoria de Extensão (PROEXT) e pela Pró-Reitoria de Pesquisa, Pós-Graduação, Criação e Inovação (PPGCI) da Universidade Federal do Recôncavo da Bahia (UFRB), utilizando-se de feiras de saúde como tecnologias educativas com a comunidade (discentes, docentes, técnicos e terceirizados) do Centro de Ciências da Saúde (CCS) e comunidade externa, especialmente do Recôncavo da Bahia, informando e construindo conhecimentos sobre os parasitos intestinais mais prevalentes encontrados em pesquisas anteriores com esta comunidade.

\section{Metodologia}

Trata-se de um estudo de natureza qualitativa, a partir do qual foi possível desenvolver e acompanhar uma atividade extensionista, compreendendo suas particularidades e os comportamentos e atitudes dos atores envolvidos, bem como sentidos e significados que atribuíram às suas experiências, procurando entendendo a interferência de fatores multiculturais sobre as situações apresentadas, e todos os possíveis significados que exercem sobre as pessoas que delas experienciam (CuestaBenjumea, 2008).

Foram realizadas duas feiras de saúde como forma de construção de conhecimentos, utilizando-se um conjunto de tecnologias, no intuito de praticar a educação em saúde.

As atividades integraram o Projeto de Extensão: “Tecnologia em saúde: metodologias ativas para o conhecimento e combate a insetos vetores de patógenos" e o Projeto de Pesquisa/Desenvolvimento Tecnológico "Tecnologia em saúde: metodologias ativas para o aprendizado na área de doenças infecciosas e parasitárias" desenvolvidos pelo Grupo de Estudos em Parasitologia Humana (GEPaH) do CCS/UFRB, constituindo-se em espaços adequados e indispensáveis para a promoção de educação em saúde com temáticas em doenças infecciosas e parasitárias, aplicando metodologias ativas, que viabilizem um aprendizado divertido e eficaz.

Foram elaboradas entre os meses de julho a dezembro de 2018 e realizadas em dezembro de 2018 e em março de 2019 com a principal temática "Prevenção de parasitos intestinais" como atividades extensionistas para devolutiva do resultado de pesquisas (Passos et al., 2020; Rossi et al., 2018) realizadas com a comunidade do CCS / UFRB na cidade de Santo Antônio de Jesus-Bahia. A pesquisa prévia tinha como objetivo, identificar os enteroparasitos presentes nesta comunidade, assim se buscou informar o público pesquisado sobre os parasitos mais prevalentes, mecanismos de infecção e formas de prevenção, ciclo de vida e sintomatologias presentes no desenvolvimento das parasitoses.

As feiras foram realizadas nos corredores do CCS e divididas em quatro estações, de forma que os participantes passassem por todas, sequencialmente, trabalhando, em cada estação, uma informação diferente e complementar. Para isso 
foram utilizados microscópios, lâminas, cartazes, notebook, maquete e jogo de tabuleiro, de acordo com as tecnologias socializadas por Santos et al. (2018) (Figura 1).

A média de participantes foi de 150 em cada uma das feiras, totalizando aproximadamente 300 pessoas que visitaram os stands e se envolveram nas dinâmicas realizadas.

Figura 1 - Panorama geral dos instrumentos utilizados na Feira de Saúde:

cartazes, maquetes, microscópios e jogo de tabuleiro - CCS / UFRB.

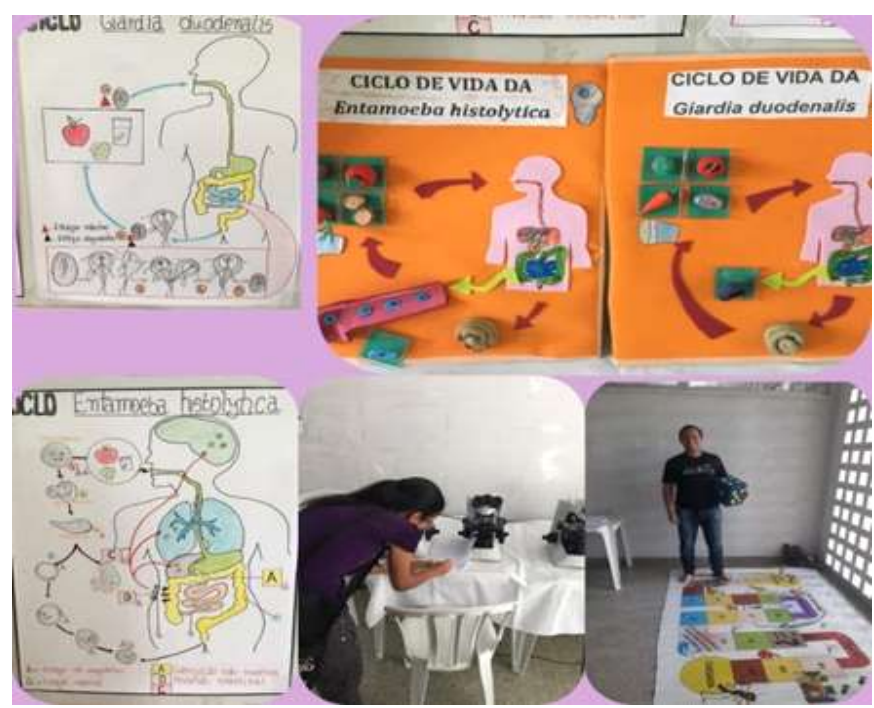

Fonte: GEPaH (2018).

\section{Resultados e Discussão}

\subsection{Relatando sobre a feira}

Na primeira estação ocorreu exposição dos resultados das pesquisas dos enteroparasitos mais prevalentes na comunidade do CCS (Passos et al., 2020), com visualização microscópica das formas evolutivas destes enteroparasitos, com destaque para os mais prevalentes, Giardia duodenalis e Entamoeba histolytica - no caso dos patogênicos e Entamoeba coli e Endolimax nana - no caso dos não patogênicos.

Na segunda estação, foram descritos os principais sinais e sintomas das infecções parasitárias, com maior destaque para a giardíase e a amebíase (Figura 2).

Ressalta-se a relevância do estudo de ações profiláticas destas duas parasitoses, como o das feiras de saúde, que se estabelecem também como profilaxia primária, procurando a partir das informações apresentadas, trabalhar medidas de prevenção. A giardíase, por exemplo, é uma parasitose intestinal amplamente distribuída pelo mundo, com alta prevalência em países em desenvolvimento, inclusive o Brasil. Embora seja uma infecção com bom prognóstico, pode apresentar gravidade em pessoas com desnutrição, fibrose cística ou algumas imunodeficiências (Santana, Vitorino, Antonio, Moreira \& Gomes, 2014). Na amebíase, o parasito E. histolytica pode invadir tecidos, originando as formas intestinal e extra- 
intestinal da doença. É mais comumente encontrada em regiões com condições econômicas deficientes. As infecções podem durar anos e podem apresentar sintomas leves, graves ou mesmo ser assintomática (Coura, 2015). Tanto na giardíase quanto na amebíase o mecanismo de infecção faz-se diretamente por mãos contaminadas, ou indiretamente por alimentos e líquidos contaminados com cistos dos respectivos parasitos.

Figura 2 - Visualização microscópica de enteroparasitos e apresentação das respectivas parasitoses - Feiras de Saúde - CCS / UFRB / Santo Antônio de Jesus - 2018/2019.
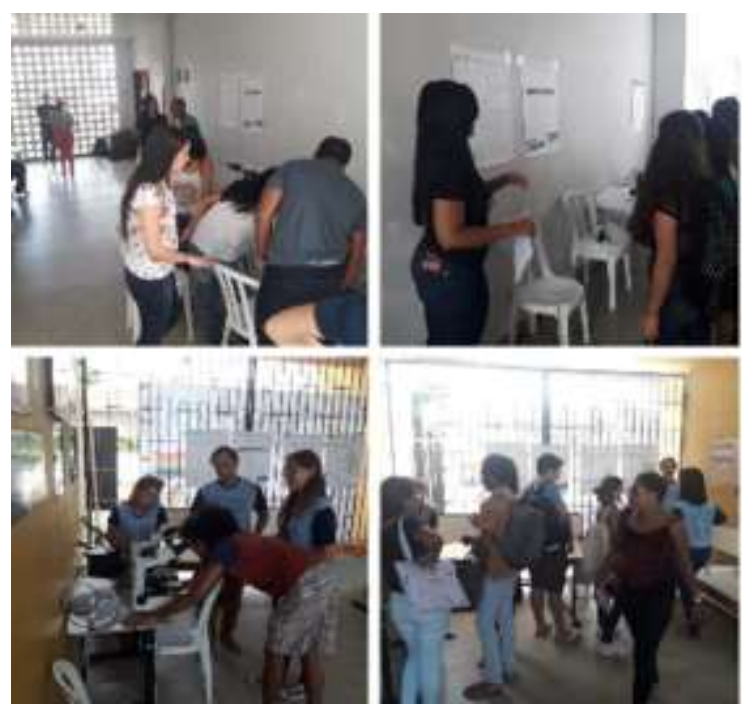

Fonte: GEPaH (2018-2019).

$\mathrm{Na}$ terceira estação, foram apresentados e discutidos os ciclos de vida dos parasitos G. duodenales e da E. histolytica, com uso de maquetes e cartazes. Nesse mesmo espaço, houve a exibição de condutas adequadas e inadequadas, que protegem ou deixam susceptíveis às infecções, sendo solicitado que os participantes colocassem plaquinhas de "curtir" (para o que costuma praticar) e "não curtir" (para o que não praticasse), trabalhando a partir daí as medidas profiláticas relativas a estas infecções (Figura 3). 
Figura 3 - Apresentação dos ciclos de vida de Giardia duodenalis e Entamoeba histolytica - Feiras de Saúde - 2018/2019.

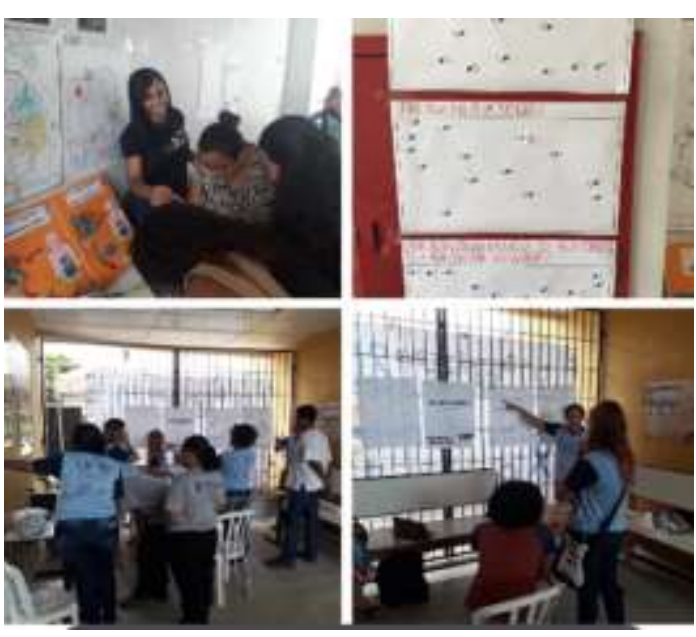

Fonte: GEPaH (2018-2019).

Na quarta estação, um jogo de tabuleiro com perguntas e respostas, baseado em Santos et al. (2018) foi utilizado para estimular a fixação do assunto abordado (Figura 4). Para a realização desse jogo foi utilizado um tabuleiro e um dado. Os participantes eram divididos em dois grupos e convidados, um de cada vez, a lançar o dado, o qual direcionava a uma pergunta. Caso o grupo acertasse, avançava a quantidade de casas indicadas no lançamento. O grupo que alcançasse a chegada primeiro era denominado como "campeão".

Figura 4 - Aplicando o jogo de tabuleiro com temas sobre os protozoários intestinais Giardia duodenalis e Entamoeba histolytica e demais enteroparasitos - Feiras de Saúde - CCS / UFRB / Santo Antônio de Jesus - 2018/2019.
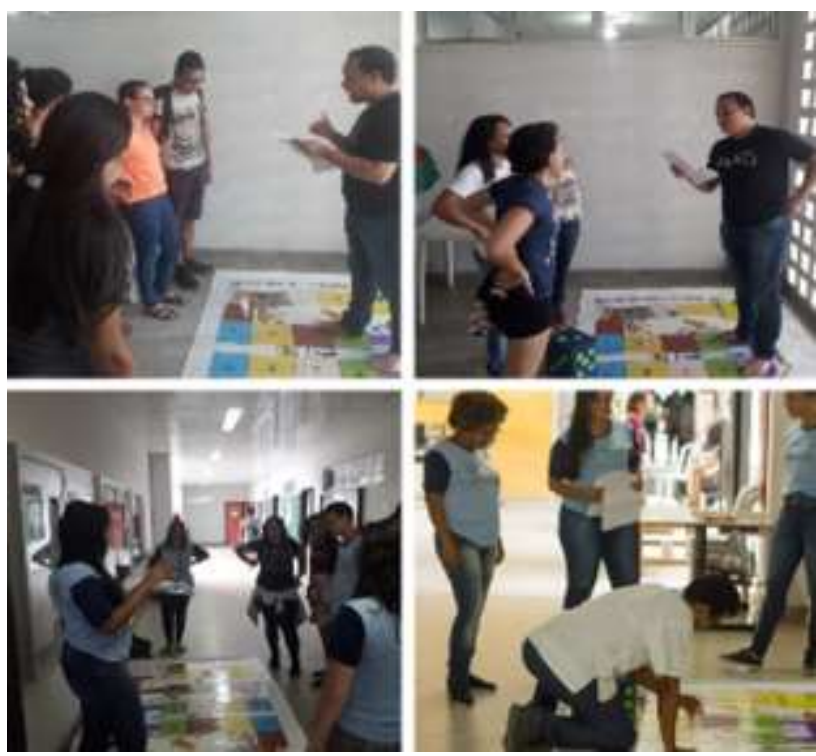

Fonte: GEPaH (2018-2019).

Além desse jogo, foi utilizado, apenas na primeira edição do evento, o Kahoot (disponível em www.kahoot.com) (plataforma baseada em jogos), como um quiz, no qual se pode adicionar perguntas que são convertidas em um jogo com pontuação, interação e ranqueamento, como traz Sande e Sande (2018). O uso dessa ferramenta permitiu estimular a 
participação, bem como testar os conhecimentos pré e pós percurso sobre a temática trabalhada (Figura 5). Sendo realizado antes dos participantes entrarem no circuito das estações (pré-teste), e logo após ter passado pelo mesmo (pós teste).

Optou-se por não utilizar este recurso na segunda edição da feira, em maço de 2019, pois a mesma estava inserida em um evento maior (o Seminário Reecôncavo Saúde e Semana Acadêmica 2019.1), que ocorre todo inicio de semestre no CCS/UFRB (Bomfim et al., 2019), com estimativa de público maior. Assim, para dinamizar a passagem dos visitantes manteve-se apenas o jogo de tabuleiro para fixar o conteúdo apreendido durante a feira.

Figura 5 - Aplicando o pré e pós teste com o aplicativo Kahoot - Feira de Saúde - CCS / UFRB / Santo Antônio de Jesus 2018.

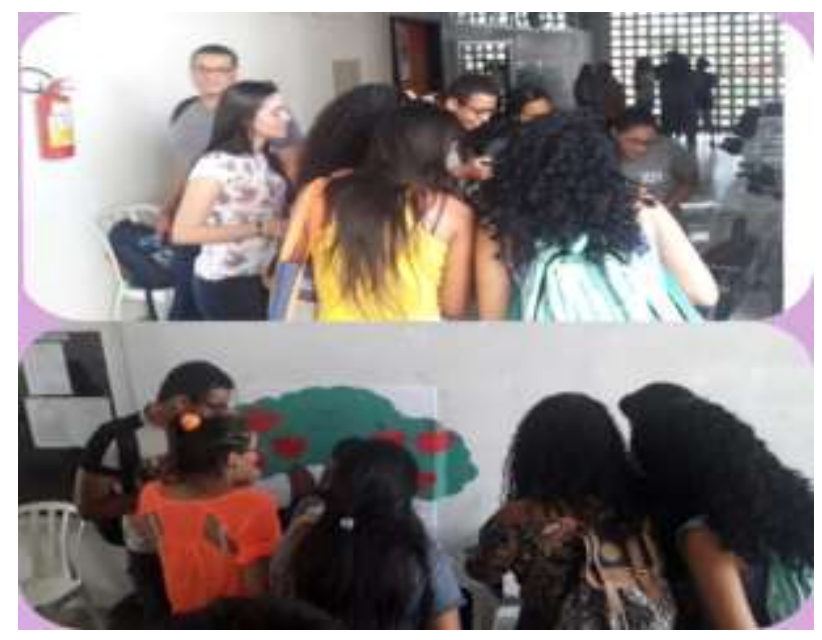

Fonte: GEPaH (2018).

\subsection{Discutindo sobre a feira}

A realização das feiras de saúde divididas em estações mostrou-se satisfatória e de extrema relevância na construção dos conhecimentos. Pois, além de ter sido um importante espaço para realização de educação em saúde, informando os participantes, especificamente para esta ação, sobre ciclos parasitários, meio de infecção e de prevenção e sintomas das enteroparasitoses mais prevalentes na comunidade, constituiu-se também como uma oportunidade para realizar a devolutiva de uma produção científica. Como disposto por Sverzut et al. (2014), a devolutiva de dados produzidos deve fazer parte do processo das pesquisas científicas, demostrando assim responsabilidade social para além da produção teórica.

E apesar de se tratar de um publico, em sua maioria de graduandos da área de saúde, pode-se perceber que algumas informações trabalhadas durante as estações das feiras, eram desconhecidas por algum participante do público visitante.

Os instrumentos utilizados também foram importantes para o desenvolvimento das feiras, pois os elementos visuais como os desenhos e as maquetes contribuíram significantemente para a compreensão dos temas abordados, instigando e estimulando os participantes a questionarem e a esclarecer duvidas, mostrando a importância de diversificar metodologias de aprendizado. Além disso, a utilização dos jogos como metodologias para ensino e aprendizagem, como afirmam Lima, Moreira e Castro (2014) permite maior adesão e interação dos participantes, além de favorecer melhor consolidação de conteúdos expostos.

Nas feiras, pode-se constatar o quanto o uso de metodologias diversificadas estimularam a participação dos ouvintes, contribuindo assim para que o processo de aprendizagem tivesse um caráter didático e emancipatório, auxiliando na sensibilização e conscientização dos sujeitos impulsionando-os e mobilizando-os a transformar seus hábitos de vida, principalmente no que se refere à higiene pessoal e alimentar. 
Ao utilizar o aplicativo Kahoot, na primeira edição da feira, como um recurso digital para avaliar o conhecimento prévio e após o percurso nas estações, pode-se reafirmar essa assertiva. Corroborando para essa assertiva, a análise do pré-teste mostrou que houve acerto de $75 \%$ das questões realizadas, enquanto que o pós teste alcançou 87,5\% de acerto. Além disso, o aplicativo permitiu constatar quão significativo e eficaz foram às informações trabalhadas, visto que o mesmo possibilitou avaliar o quanto se compreendeu do exposto durante as feiras. Podendo-se assim, refletir sobre a modificação do conhecimento, devido à adição de novos dados.

A aplicação de instrumentos com finalidades de "pré" e "pós teste" podem ser considerados efetivos no processo de aprendizagem significativa. Como afirmam Castro e Moreira (2001), a utilização desse tipo de metodologia permite que haja uma somatória dos significados e do novo conhecimento, proporcionando assim a diferenciação do conhecimento prévio. Destarte, a utilização do pré e pós testes, através dos jogos, contribui para construção de conhecimento não mecânico, visto que os ouvintes eram estimulados a organizar, incorporar, compreender e fixar as novas informações.

Os produtos propostos, confeccionados (Santos et al., 2018) e trabalhados nas feiras se figuraram como tecnologias educativas, como facilitadores da aprendizagem. Empregá-los, no contexto educacional proposto, apresentou diversas dificuldades, como o desafio de juntar harmoniosamente as tecnologias com o conteúdo trabalhado e dos envolvidos (pesquisadores, extensionistas e participantes ouvintes) aceitá-las como educacionalmente válido.

Estas ações apresentaram tecnologias que, unidas aos hábitos dos envolvidos, possibilitam potencializar seus conhecimentos em doenças infecciosas e parasitárias, unindo a tecnologia com a informação correta e devidamente direcionada para gerar uma postura crítica nos mesmos. Sensibilizando, conscientizando e mobilizando os membros da comunidade CCS para o enfrentamento de situações individuais e coletivas que interferem na qualidade de vida, no que diz respeito às infecções parasitárias intestinais, assimilando as noções sobre prática de cuidados preventivos e hábitos saudáveis associados aos saberes e hábitos populares trabalhados nas dinâmicas propostas.

Procurou-se por meio destas atividades trabalhar a popularização da ciência, tornando-a acessível para diversos públicos, disseminando-a de forma democrática, aproximando simultaneamente o saber científico da sociedade e vice-versa (Coelho, Silva, Tamiasso-Martinhon \& Sousa, 2020). A professora Aline Lima em Souza (2021, p. 11) relata que as trocas de saberes entre os campos educação comunitária e saúde promovem a democratização do saber acadêmico e o retorno deste saber à universidade reelaborado.

É importante ressaltar que estas atividades, inseridas no PIBEX e no PIBITI, programas institucionais da UFRB, representam uma oportunidade para o discente extensionista e para o discente pesquisador, em conjunto com sua orientadora e demais membros das equipes de trabalho, buscarem uma formação pautada na interação entre a universidade e a comunidade, desenvolvendo ações junto a esta, atentando-se sempre para as necessidades sociais, qualificando assim suas práticas. Foi nítido o envolvimento dos graduandos em saúde nas diferentes etapas do evento: da definição dos temas, elaboração das dinâmicas, até o momento da apresentação da atividade aos públicos. Infere-se, que ações deste tipo, como Feira de Ciências, Feira de Saúde, Feira de conhecimentos e afins, são relevantes para a promoção da educação científica e em saúde, seja para público infantil e/ou demais faixas etárias, contribuindo para mudanças positivas no ambiente escolar/acadêmico/comunitário, assim como, um meio de inclusão social, podendo adaptar-se quando da necessidade do público de ação (Passos et al., 2019; Pereira, Alves, \& Coutinho-Silva, 2020). 


\section{Considerações Finais}

Este relato apresentou uma inovação na metodologia do ensino em saúde de relevância no contexto da extensão. As metodologias utilizadas, além de promover a valorização do saber sobre as parasitoses mais prevalentes no grupo estudado, corroboraram para a construção de conhecimentos dos participantes, possibilitando a transformação dos hábitos inadequados que possibilitavam a tríade epidemiológica da infecção (permitindo o encontro do parasito com o hospedeiro no ambiente). A participação ativa dos ouvintes nessa ação em saúde, mostrou-se também efetiva na construção destes conhecimentos.

Ao finalizar as feiras foi nítida a percepção de que por mais simples que sejam as ações de educação em saúde, estas quando bem realizadas, podem surtir efeitos significativos, e alcançar os objetivos de seus desenvolvimentos. Além disso, o processo de construção das feiras, auxiliou no desenvolvimento de habilidades e técnicas necessárias ao futuro profissional da saúde, estimulando assim o exercício da criatividade e oralidade.

Nesse sentido, tanto o PIBEX quanto o PIBITI, exercem grande importância na formação de discentes qualificados, pois abrem espaços para que esses futuros profissionais se desenvolvam, buscando estratégias para trabalhar com público e promover ações com qualidade e com criatividade para abordar temas em saúde.

Para trabalhos futuros, há perspectivas de que essas ações sejam realizadas em outros espaços escolares e/ou comunitários, porém, considerando-se a pandemia de COVID-19, há uma expectativa do grupo de autores em realizar a atividade a distância, em ambientes virtuais, por aplicativos digitais, adequando-a a este propósito, sem a interação presencial, utilizando-se de ferramentas digitais em plataformas específicas, agregando conhecimento em saúde para públicos diversos e em diferentes localidades do estado da Bahia ou demais cidades do Brasil.

\section{Referências}

Bomfim, E. S., Carvalho, K. S., Santos, R. S.; Santos, E. R., Jr.; Rangel, M. S.; Amor, A. L. M. (2019). Reencôncavo Saúde: atividade extensionista em um Centro de Ciências da Saúde. Anais do $8^{\circ}$ Congresso Brasileiro de Extensão Universitária. Natal - RN: SEDISUFRN, 1756 - 1757.

Brasil (2009). Temático promoção da saúde IV. Brasília (DF): Ministério da Saúde (BR). Organização Pan-Americana da Saúde.

Coelho, F. J. F., Silva, S. M., Tamiasso-Martinhon, P., \& Sousa, C. (2020). Popularização da ciência, educação popular e ensino de ciências e saúde a partir do voluntariado: potencialidades e limitações no projeto PEP Ciências na visão dos monitores. Rev. Ed. Popular, 19(3), $273-292$.

Coura, J. R. (2015). Dinâmica das Doenças Infecciosas e Parasitárias. $2^{\text {a }}$ ed. Rio de Janeiro: Guanabara Koogan.

Cuesta-Benjumea, C. (2008). Por dónde empezar? la pergunta en la investigación cualitativa. Enferm Clin. 18(4), $205-210$.

Ferreira, M. L. S., Moura, J. F. L., Silva, E. S., Rocha, R. F., Olivares, A. I. O., \& Hayd, R. L. N. (2010). Feira de saúde do curso de medicina da UFRR: uma aproximação com a comunidade. Rev Bras Educ Med., 34(2), 310-4.

Filatro, A., \& Cavalcanti, C. C. (2018). Metodologias inov-ativas na educação presencial, a distância e corporativa. 1. ed. São Paulo: Saraiva.

Lima, L. F., Moreira, O. C., \& Castro, E. F. (2014). Novos olhares sobre o ensino da Fisiologia Humana e da Fisiologia do Exercício. RBPFEX-Revista Brasileira de Prescrição e Fisiologia do Exercício, 8, 47.

Maike, V. R. M. L. (2013). Jogos educacionais tipo RPG: design e desenvolvimento contextualizados no laptop XO. Dissertação (mestrado) - Universidade Estadual de Campinas, SP: [s.n.].

Matta RD. Você tem cultura? Jornal Embratel [online]. 2009 [acesso 2011 Mai 11]; http://naui.ufsc.br/files/2010/09/DAMATTA_voce_tem_cultura.pdf

Costa, S. S. C., \& Moreira, M. A. (2001). A Resolução de Problemas como um tipo especial de Aprendizagem Significativa. Cad. Cat. Ens. Fís., 18(3), 263277.

Passos, A. S., Santos, E. R., Jr., Bomfim, E. S., Carvalho, K. S., Matos, P. J. S., \& Amor, A. L. M. (2019). Feira Interativa: utilização de metodologias ativas no processo de educação em saúde. Anais da V Reunião Anual da Ciência, Tecnologia, Inovação e Cultura no Recôncavo da Bahia / V RECONCITEC, Cruz das Almas, 813.

Passos, A. S., Santos, E. R., Jr., Andrade, R. S., Bomfim, E. S., Ribeiro, L. S., \& Amor, A. L. M. (2020). Encontro de parasitos em uma comunidade acadêmica correlacionado com locais de origem e de residência dos participantes. Anais da VI Reunião Anual da Ciência, Tecnologia, Inovação e Cultura no Recôncavo da Bahia / VI RECONCITEC. 434. 
Research, Society and Development, v. 10, n. 6, e54910616168, 2021

(CC BY 4.0) | ISSN 2525-3409 | DOI: http://dx.doi.org/10.33448/rsd-v10i6.16168

Pereira, G. R., Alves, G. G. H. V. S., \& Coutinho-Silva, R. (2020) Educação Científica nos anos iniciais do Ensino Fundamental por meio da Feira de Ciências dos Pequenos Cientistas. Research, Society and Development, [S. l.], 9(7), e990975140. DOI: 10.33448/rsd-v9i7.5140. https://rsdjournal.org/index.php/rsd/article/view/5140.

Rossi, R. C., Andrade, R. S., Albuquerque, W. A., Lima, L. B. A., Santos, G. A., \& Moreno-Amor, A. L. (2018). Aspectos demográficos, socioeconômicos e culturais associados à infecções enteroparasitárias em uma comunidade acadêmica. Anais do XII Seminário Estudantil de Pesquisa, Inovação e Pós-Graduação e III Simpósio de Extensão (XII SEPIP), 130

Salci, M. A., Maceno, P., Rozza, S. G., Silva, D. M. G. V., Boehs, A. E. \& Heidemann, I. T. S. B. (2013). Educação em saúde e suas perspectivas teóricas: Algumas reflexões. Texto Contexto Enferm., Florianópolis, 22(1), 224-30.

Sande, D., \& Sande, D. (2018). Uso do Kahoot como ferramenta de avaliação e ensino-aprendizagem no ensino de microbiologia industrial. HOLOS, 1, 34.

Santana, L. A., Vitorino, R. R., Antonio, V. E., Moreira, T. R., \& Gomes, A. P. (2014). Atualidades sobre giardíase. JBM, 102(7), 7-10.

Santos, E. R., Jr., Carnevalli, L. M., Mota, L. H. S., Santos, R. S., Rossi, R. C., Alves, J. V. V., \& Amor, A. L. M. (2018). Tecnologias educativas como instrumentos para o conhecimento e combate de agentes de doenças infecciosas e parasitárias. In: Salgado, Y. C. S. (Org), Patologia das doenças, Ponta Grossa (PR): Atena Editora, 3, 161-173

Souza, S. (2021). Inserção comunitária em saúde é prática pedagógica curricularizada na UFRB. Revista Extensão, ed. 19, 2, 10-13.

Sverzut, C., Lucchese, R., Vera, I., Santana, F. R., Evangelista, R. A., Fernandes, I. L., Bueno, A. A., \& Castro, P. A. (2014). Devolutiva dos resultados de pesquisa com grupos em equipe de saúde de família. Enciclopédia Biosfera, Centro Científico Conhecer, 10(19), 212-225.

Tavares, C. Z., Costa, L. S., Ninahuaman, M. F. M. L., Marques, G., \& Santos, D. C. (2017). Educação em saúde por meio de feiras. REFACS, 5(2), $245-254$. 